\title{
Preventive Safety: Warning System for Control Loss of Two-Wheeled Vehicles
}

\author{
Hamid Slimi*, Hichem Arioui*, Lydie Nouveliere* and Saïd Mammar*. \\ *Informatics, Integrative Biology and Complex Systems, \\ 40 Rue de Pelvoux, 91020 Evry Cedex, France. \\ Hamid.Slimi@iup.univ-evry.fr
}

\begin{abstract}
The present study aims at identifying existing and emerging Intelligent Transport System (ITS) that have the possibility to enhance the safety of motorcycle rider. A review of the literature revealed that very few commercially available ITS currently exist specifically for motorcycles, although several emerging technologies were identified. Consultations with international experts in ITS, motorcycle safety, motorcycle manufacturers and various road safety research organizations confirmed this report. However, there are emerging and existing technologies for other vehicles that have the potential to address key motorcycle safety issues.

In spite of the emergence of active and passive safety, these systems in most cases do not avoid the accident.

The purpose of this paper is to provide assistance to the rider by incorporating a warning system for accident situations, which can alert the driver sufficiently in advance so that he can anticipate the accident.

Index Terms - Motorcycle dynamics, Rider assistance, Simulator, Motorcycle safety, Warning system, Intelligent transport systems, Roll dynamics.
\end{abstract}

\section{INTRODUCTION}

$S$ ince 2002, the French government decided to make fight against the road accidents, one of the three large programs of the last five year. In spite of encouraging results obtained since the use of speed control systems, the motorcycle remains a particularly dangerous mode of transport: the number of death is still very high, and if one takes into account the number of travelled kilometers, the risk of death for a motorcycle rider is 21 times higher than that for other drivers.

During the last twenty years, passengers cars experienced several advances in passive and active safety systems. Cars today are almost all equipped with one or several airbags and ABS systems. More powerful systems such as ESP, brake assist systems and traction control systems or belt tensioners are becoming quickly democratized.

On the other hand, during the same period, the backwardness taken by motorcycles is continually growing. For example, the ABS, which exists since nearly 15 years, is still reserved to few top range models. The braking distributor is less expensive but still remains marginal on the whole of the market. Motorcycle airbags, whose development seems difficult, does not come to be imposed. The recent braking amplifier seems to be promising, but its diffusion remains for the moment very restricted.

Our study, motivated by this fact, aims at partly overcoming these deficiencies. Its objective relates into the three following aspects :

- To acquire the knowledge on the behavior of the two wheeled vehicles in dangerous situations,

- To prove the feasibility of an onboard system which can be able to detect critical situations,

- To design a demonstrator of this onboard system in order to warn the rider and/or to activate passive safety systems.

Thus, the purpose of this paper is to provide preventive assistance to the rider by incorporating a warning system for accident situations.

This paper is organized as follows : in the next section, results of an accidentology study are provided; some relevant cases of control loss are detailed. Sections 3 will give an overview of commercial systems offered by manufacturers and research projects in this field. Then, a talk about risk indicators in longitudinal and lateral motion was conducted. In section 6 , a description of the dynamic model for the rider-motorcycle system and some relevant characteristics are presented. Tire road interaction model is highlighted. In section 7 , the simultaneous stabilization approach is presented. Controllers are synthesized using an 
$L Q G$ (Linear Quadratic Gaussian) performance index. In section 8, we present a method for generating alert. Finally, we present the prototype underway of realization. The last section is devoted to the conclusions, and the major parameters used in this paper are listed.

\section{ACCIDENTOLOGY CONTEXT}

A recent accidentology study revealed that more than $70 \%$ of urban and sub-urban motorcycle accidents occur at a speed lower than $50 \mathrm{~km} / \mathrm{h}$ $[10,9]$. It is also shown that $15 \%$ of the accidents happen alone. When another vehicle is involved during the accident, the rider is responsible for $37 \%$ of the cases and the adverse vehicle in $50 \%$ of the cases. The remaining $13 \%$ are due to bad weather conditions or infrastructure failure.

The study of 200 [12] reported motorcycle accidents leads to the isolation of 47 cases of control loss due to the incompatibility between the rider and the machine leading to control loss [12]. This incompatibility is originated either by the own overestimation of the rider or motorcycle performance or by bad interpretation of the environment. It was noticed that for $75 \%$ of the accidents, the rider attempts a braking or an avoidance emergency maneuver.

\section{AUTOMATION AND EMBEDDED ELECTRONICS}

The embedded electronics has not finished revolutionizing the automobile [12]. Electronic equipment roughly represents $20 \%$ of the manufacturing cost of a vehicle and reaches $35 \%$ for high-end vehicles equipped with a guidance system. The role of these devices ranges from low level (to control the operation of mechanical functions) to the highest (to improve safety and comfort of the driver). These functions are either automated features such as speed regulation (ACC: Adaptative Cuise Control), braking system of controlled vehicles (ABS: Anti-lock Brake System) or driving aid functions. Despite of the delay of two-wheeled vehicles compared to standard vehicles, registered in this area, the last years, there has been an incredible technological development.

\section{PROPOSED ARCHITECTURE FOR THE WARNING SYSTEM}

The goal of this section is to present a proposal for assistance to drivers of two-wheeled (Motorcycles and Scooters) by preventing them sufficiently in advance to anticipate a dangerous situation (Figure. 1).

The overall objective is to study and develop systems for motorcycle safety through a preventive detection of the accident. This study will result in the realization of a prototype (vehicle test). For the development of the safety system, we have to:

- Learn about the behavior of two-wheelers in situations before the accident,

- Prove the feasibility of embedded electronic systems able to detect a critical situation on board,

- Design an embedded simulator to alert the driver and trigger safety active and/or passive systems.

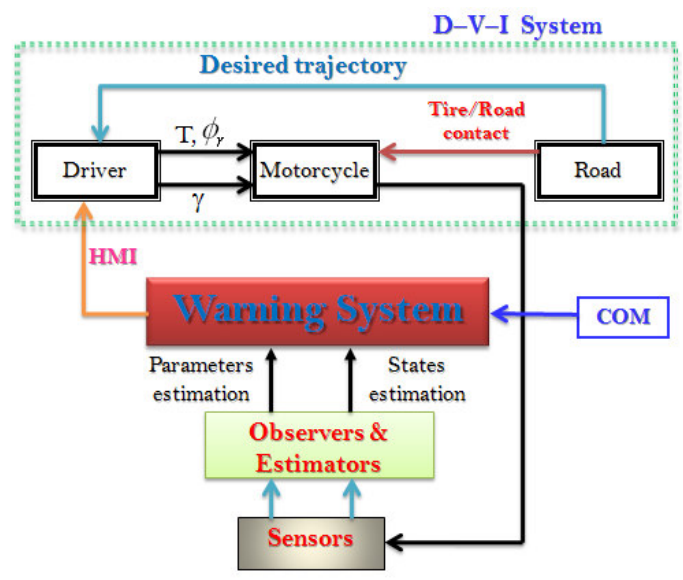

Figure 1. Architecture of the warning system

The warning system takes into account :

$\checkmark \quad$ Driver behavior: represented by the longitudinal and lateral mobilized adhesion.

$\checkmark$ Geometry of the road : represented by slope, curvature...

$\checkmark$ Motorcycle Dynamics : represented by its center of gravity, contact tire/road, external forces...

The warning system is in interaction with the triplet D-V-I via a HMI.

In the triplet D-V-I, the driver influences the motorcycle dynamics by its inputs (Torque, roll and 
lean angles), the road also affects the vehicle through the tire/Road contact, and advises the desired trajectory to the driver.

The warning system exploits the information given by different physical sensors, for knowing the dynamics states evolution and dynamics parameters variation. However, these states and dynamic parameters are not directly measurable: sensors do not exist yet, or they are prohibitively expensive.

To address these constraints, software sensors based on the criterion of states observation exist. These software sensors often called "observers" can reconstitute the state vector in real time, from knowledge, on an interval of time, of system inputs, measurements output by sensors relatively cheaper and the knowledge of model.

\section{THE RISK INDICATORS}

\section{a. Indicators for the lateral mode}

A first group of indicators is directly linked to the proprioceptive dynamics of the vehicle :

- The lateral acceleration, directly obtained from an accelerometer.

- The yaw rate, combined with speed and the longitudinal wheel angle is indicative of an effect of oversteer or understeer conditions. These elements, also like the lateral acceleration, are available as standard on a motorcycle equipped with $\mathrm{ABS}$ and ASC.

- The lateral speed. This speed is not directly measurable; its estimation is possible from other measures.

A second group of indicators requires the presence of exteroceptive sensors :

- The lateral movement of the vehicle with respect to the center of the track. This indicator can be easily obtained by a video sensor as a front vision.

- The Time to Line Crossing (TLC), which represents the time needed, given the speed of the vehicle, to cross the edges of the track. This indicator combines dynamic vehicle location on the track and the road geometry is very useful, but not easy to compute.

\section{b. Indicators for the longitudinal mode}

In the case of one vehicle only (speed regulation), the two primary indicators are: the steady longitudinal speed and acceleration. During vehicle following, the driver simultaneously manages vehicle speed and the spacing. Several indicators exist:

- The spacing, compared to the minimum spacing so called safety distance.

- The relative speed, normally null.

-The time to collision.

- The headway time.

\section{MOTORCYCLE DYNAMIC MODELLING}

The aim of this section is to provide general characteristics of the dynamic model of the lateral motion of the motorcycle including the roll motion. Used formula for tire-road forces interactions make use of a modified Pacejka formula of [4] and [19] in order to include the influence of the camber angle. Finally, a roll feedback rider leaning movement is added to that model reproducing the rider upper body actions aiming at stabilizing the motorcycle.

\section{a. Lateral motion modelling}

In comparison, to vehicle modelling, the literature in motorcycles modelling is less widespread. Since the two rigid bodies modelling proposed in [1], up to seven bodies and more complex models have been studied [2]. The complexity was generally made necessary for the study of some specific behavior during acceleration, braking or steering phases [3,5,6]. The model presented in this paper has four jointed rigid bodies and is inspired from those presented in [7,8]. It includes the driver upper body lean motion. As in [7], the four rigid bodies of this driver-motorcycle are as following (Figure 2):

- Front fork assembly: front fork, handle bar, front wheel and shock absorber.

- Rear frame: engine rotating in transverse axis, chassis, the lower body and legs of the rider.

- Rear wheel.

- Rider upper body: the upper body of the rider including hands and head.

This model can be reduced to only three rigid bodies by putting the rear wheel body in the rear frame. In this case, it is no more possible to model the twisting movement at the rear swinging arm. 
TABLE 1: Masses

\begin{tabular}{|l|l|l|}
\hline Parameter & Value & Description \\
\hline $\mathrm{M}$ & 285.5 & Total mass \\
\hline $\mathrm{m}_{\mathrm{f}}$ & 40.5 & Front fork mass \\
\hline $\mathrm{m}_{\mathrm{r}}$ & 170 & Rear part mass \\
\hline $\mathrm{m}_{\mathrm{b}}$ & 25 & Rear wheel mass \\
\hline $\mathrm{m}_{\mathrm{p}}$ & 50 & Upper body mass \\
\hline
\end{tabular}

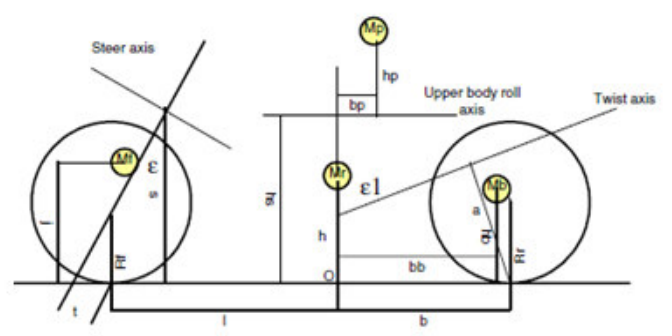

Figure 2. Motorcycle bodies and dimensions.

The caster angle of the front fork is equal to $21^{\circ}$. Relevant distances are shown on Figure 2, other model data, including mass, are shown in Table I. The obtained nonlinear model has 8 state space variables given by

$$
x=\left[\nu, \dot{\phi}, r, \dot{\delta}_{f}, \phi, \delta_{f}, f_{f}, f_{r}\right]^{T}
$$

where $v$ is the lateral speed, $r$ is the yaw rate, $[\dot{\phi}, \phi]$ are respectively the roll rate and the roll angle and $\left[\dot{\delta}_{f}, \delta_{f}\right]$ are respectively the steering angle rate and angle value. The forces $f_{f}$ and $f_{r}$ are the front and lateral forces of rear tires. Equations of motion are obtained by gathering the lateral motion equation, the yaw equation, the roll equation, the front fork hinge equation and finally the two front and rear tire forces relaxation equations. The model presents 2 separated inputs given by the rider control torque $T$ and the rider lean motion whose input is the relative roll angle $\phi_{r}$. The whole control vector is $\left[T, \phi_{r}\right]$

Beyond the classical mechanical equation, the most important component of the model is the tireroad interaction module. The front and rear tire lateral forces are function of the sideslip angle at the tire-road contact location and the camber angle.

\section{b. Tire road interaction}

We used the Pacejka model to represent the efforts exerted on each tire. This model is based on the mathematical representation of the tire dynamic behavior using analytical functions having a particular structure.
Lateral forces of front and rear tires are function of the sideslip angle $\alpha_{i}$ at the tire-road contact location and the camber angle $\gamma_{i}$. Here, the index $i$ stands for $f$ (front) or $r$ (rear). In this paper, the magic formula of Pacejka is used for each tire in order to determine the lateral forces:

$$
f_{i}\left(\alpha_{i}, \gamma_{i}\right)=d_{i}\left(\gamma_{i}\right) \sin \left[\begin{array}{l}
c_{i} \tan ^{-1}\left(b_{i}\left(\gamma_{i}\right)\left(1-e_{i}\right) \alpha_{i}\right. \\
+e_{i} \tan
\end{array}\right.
$$

The coefficients $b_{i}, c_{i}, d_{i}, e_{i}$ depend on the tire characteristics, on the road conditions, and on the vehicle operational conditions. Parameter values, when road friction is high and camber angle is zero, are given in Table 2 .

Table 2 : Tire model parameters on high friction road

\begin{tabular}{|c|c|c|c|c|}
\hline Tire & $\mathbf{b}_{\mathbf{i}}$ & $\mathbf{c}_{\mathbf{i}}$ & $\mathbf{d}_{\mathbf{i}}$ & $\mathbf{e}_{\mathbf{i}}$ \\
\hline Front & 14.2574 & 1.25095 & 1010.0 & -1.661 \\
\hline Rear & 13.9481 & 1.10095 & 1835.8 & -1.542 \\
\hline
\end{tabular}

Let $\mu$ be a common road adhesion coefficient with $\mu=0.2$ for icy road and $\mu=1$ for nominal road adhesion. The effect of the road adhesion on the lateral forces is incorporated in the magic formula by changing $b i$ to $(2-\mu) \cdot b i, c_{i}$ to $\left(\frac{5}{4}-\frac{\mu}{4}\right) \cdot c_{i}$ and $d_{i}$ to $\mu . d_{i}$. In addition, the influence of the camber angle is handled by adapting the formulas for $b_{i}$ and $d_{i}$

$$
\left\{\begin{array}{l}
\mu \cdot d_{i} \rightarrow \mu \cdot d_{i}\left(1-\frac{2\left|\gamma_{i}\right|}{\pi}\right)^{2} \\
(2-\mu) \cdot b_{i} \rightarrow(2-\mu) \cdot b_{i} \cdot\left(1-\frac{\left|\gamma_{i}\right|}{4 \pi}\right)
\end{array}\right.
$$

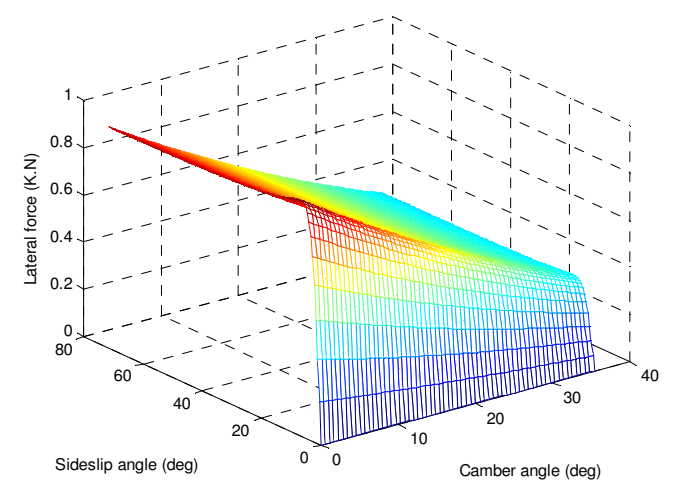

Figure 3. Front lateral force as a function of the sideslip angle and the camber angle.

The figure 3 provides a 3D plot of the front tire lateral force as a function of the sideslip angle and the camber angle. The increase of the camber angle leads to a reduction of the lateral forces. 


\section{MODEL STABILISATION}

At forward speed of $23 \mathrm{~m} / \mathrm{s}$ [13], the motorcycle is stable; however it is unstable for a large range of forward speed. The stability is only obtained around the speed of $20 \mathrm{~m} / \mathrm{s}$.

The model system motorcycle-rider is unstable; the control by stabilizing command is synthesized in the next section using a $\mathbf{L Q G}$ control law (figure. 4).

\section{a. LQG Controller}

To ensure a robust monitoring trajectory as a reference, and rejecting disturbance, we apply $\boldsymbol{L} Q$ control on the Motorcycle-rider system.

The $\boldsymbol{L} \boldsymbol{Q}$ control is an optimal feedback control which minimizes the quadratic criterion, given by:

$J=\frac{1}{2}\left\{\int_{t_{0}}^{t_{1}}\left[x^{T}(t) Q(t) x(t)+u^{T}(t) R(t) u(t)\right] d t+x^{T}\left(t_{1}\right) S x\left(t_{1}\right)\right\}$

The objective of the linear quadratic $(\boldsymbol{L Q})$ [4] is to formulate the problem of order by returning a state in terms of optimization of a quadratic criterion, which reflects a compromise between the effort required to control (actuators) and constraints we want to impose to state.

Either, system represented by its state equations and initial conditions, minimizing the criterion $\mathrm{J}$, the control law is given by the following relationship:

$u_{\text {opt }}(t)=-F(t) x(t)=-R^{-1}(t) B^{T}(t) P(t) x(t)$

The $\boldsymbol{L} \boldsymbol{Q}$ control [4] assumes that all its states are available to the measures, which is not the case in practice, hence the need for the $\mathbf{L} \boldsymbol{Q G}$ that combines a feedback control $\boldsymbol{L} \boldsymbol{Q}$ and with Kalman filter.

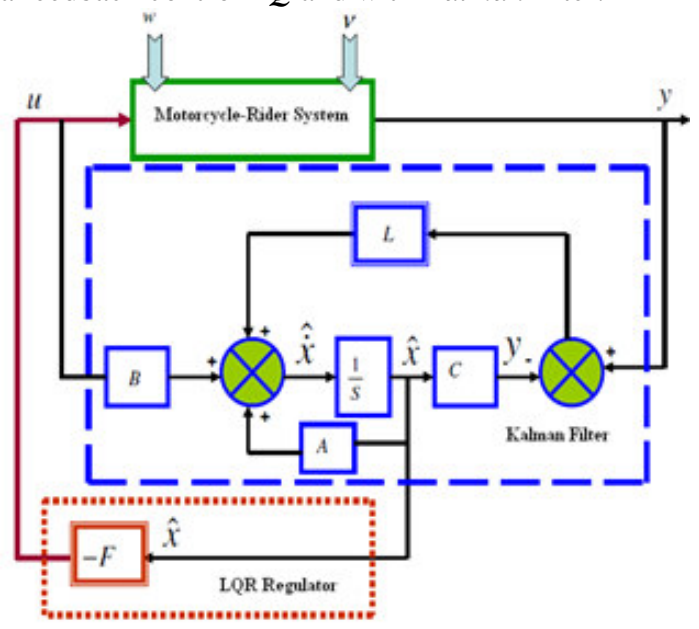

Figure 4. LQG control architecture.

\section{b. Simulation results}

We simulate a maneuver type chicane given on the figure 5 , on the human applied torque.

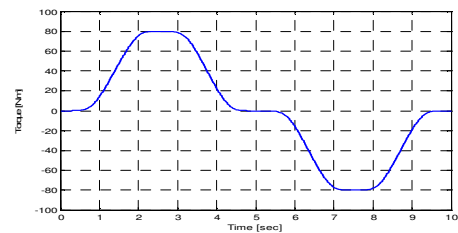

Figure 5. Steering torque.

Figures: 6, 7, 8, 9, 10, 11, show the response of motorcycle-rider in: lateral speed, yaw rate, roll rate, steering rate, roll angle and steering angle.

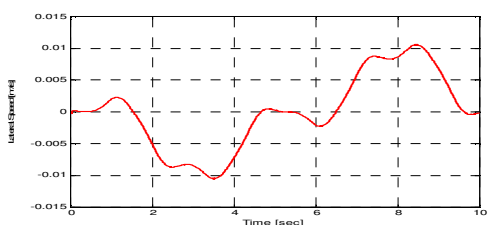

Figure 6. Lateral speed.

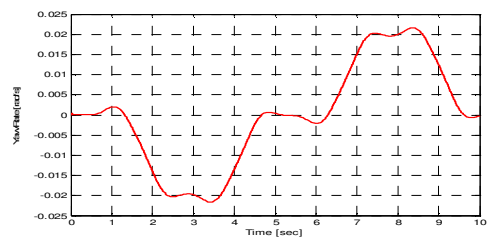

Figure 7. Yaw rate.

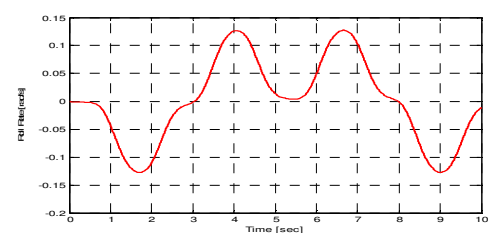

Figure 8. Roll rate.

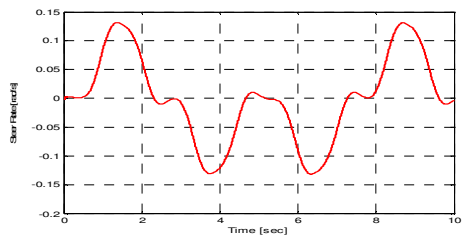

Figure 9. Steer rate.

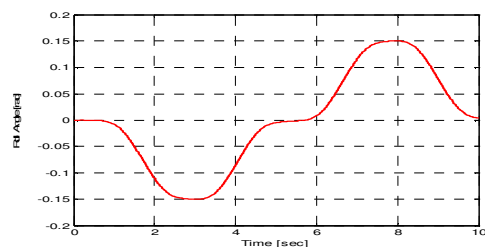

Figure 10. Roll angle. 


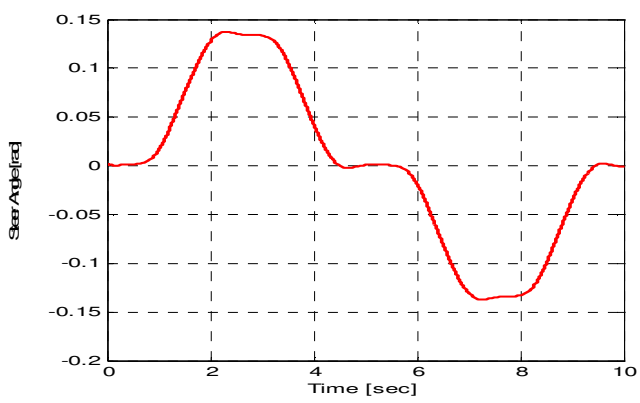

Figure 8. Steer angle.

The presented simulation results show the ability of the control to ensure proper monitoring of the reference signal of the system with good performance.

\section{WARNING GENERATION}

The original principle detection of a dangerous situation (the accidents), that we have established, is the following : from the angle of the handlebars, we will rebuild, using a model of vehicle, the nominal corresponding yaw angle $\mathrm{y}_{\text {nom. }}$ In nominal, we consider a normal situation, without beginning or slip sliding for this construction. We then assess the degree of correlation between the angle $\mathrm{y}_{\text {nom }}$ rebuilt and the yaw angle measured by the rate gyros of inertial unit.

Once this degree of correlation exceeds a certain threshold, the situation is said dangerous.

The Roll-over is, however, characterized by the passage of the motorcycle upright, where the issues of vertical and lateral accelerometers are $g_{z}=g$ (constant of gravity) and $\mathrm{g}_{\mathrm{y}}=0$, to the motorcycle in a prone position where $\mathrm{g}_{\mathrm{z}}=0$ and $\mathrm{g}_{\mathrm{y}}=\mathrm{g}$.

An indicator of fall corresponds to the conditions: $\left|g_{z}\right|<1 \mathrm{~m} / \mathrm{s} 2$ and $\left|g_{y}\right|>9 \mathrm{~m} / \mathrm{s}^{2}$. Finally, an indicator of future fall (future accident) is given by the conditions: $\left|g_{z}\right| \leq 5 \mathrm{~m} / \mathrm{s}^{2}$ and $\left|g_{y}\right| \geq 5 \mathrm{~m} / \mathrm{s}^{2}$, the case of equality corresponding to a roll angle equal to 60 degrees.

\section{ADOPTED VALIDATION STEP}

This section aims at validating the simulation model developed above and a warning system. The equipped motorcycle is an Electric Scooter Peugeot model (figure 12). This motorcycle is mainly equipped with sensors which measure the angular velocities, translational accelerations, steering angles and also wheel speeds.

Angular velocities and accelerations are obtained by use of central unit. These sensors are commonly used in motorcycle world such as in ASC systems. The range of the gyroscope is of $100 \% \mathrm{sec}$ and the Corriolis acceleration is $\pm 3 g$. These resolutions appear as realistic for our application. To obtain accelerations and an angular rate in all directions we use a central unit as shown on figure 12 .

The purpose now is to validate the model developed and the warning system (underway).

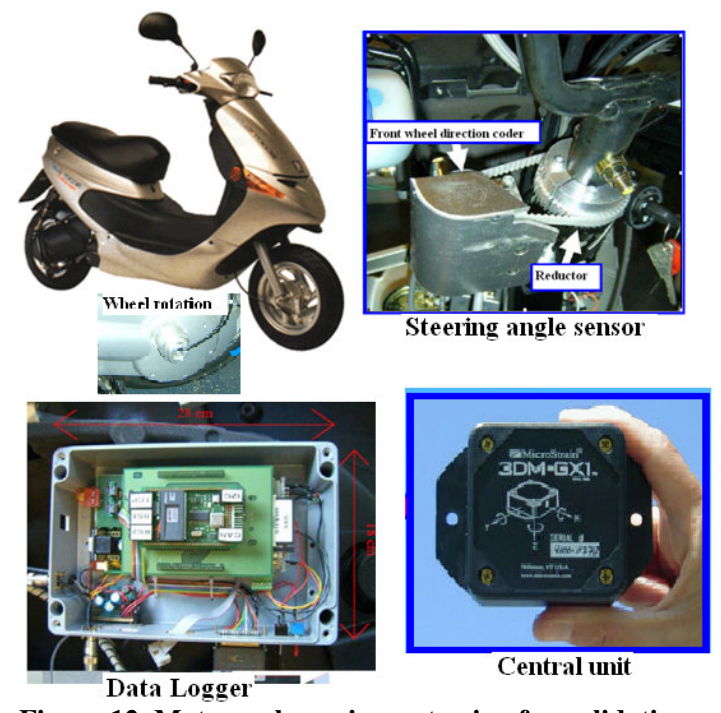

Figure 12. Motorcycle equipment using for validation.

\section{APPENDIX A: NUMERICAL VALUES}

$a=L-b=0.87 \mathrm{~m}, \quad a_{r}=0.14 \mathrm{~m}, \quad L=1.4 \mathrm{~m}$, $b=0.53 \mathrm{~m}, c=0.04 \mathrm{~m}, c_{1}=0.12 \mathrm{~m}, e=0.37 \mathrm{~m}$, $f=0.70 \mathrm{~m}, h=0.65 \mathrm{~m}, \quad h^{\prime}=0.82 \mathrm{~m}, h_{b}=0.92 \mathrm{~m}$, $h_{r}=0.79 \mathrm{~m}, h_{v}=0.62 \mathrm{~m}, h_{1}=0.82 \mathrm{~m}, h_{1}^{\prime}=0.7 \mathrm{~m}$, $k=-c=-0.04 \mathrm{~m}, \varepsilon=21 \mathrm{deg}$.

\section{CONCLUSIONS}

We presented in this paper an overview of research on different assistance for the twowheelers. The research, though limited compared to the four wheeler, are launched and results in different applications are very promising. But car manufacturers remain cautious in introducing preventive safety features, because, in addition to issues of responsibility, aspects of sharing and coupling with the driver are far from controlled.

General aspects of a motorcycle model are presented. Some stability aspects are discussed. A control synthesis procedure is developed for the stabilization of the motorcycle using the $\boldsymbol{L} \boldsymbol{Q}$ controller.

We saw that the preventive safety offers an interesting alternative; in this kind of assistance the driver can be warned in time by the warning system to anticipate potentially dangerous situations. An architecture of the warning system was proposed. 


\section{REFERENCES}

[1] R.S. Sharp,"The stability and control of motorcycles," Jour. Mech. Eng. Sci. 13(5), 316-329, 1971.

[2] R.S. Sharp, and D.J.N. Limebeer,"A motorcycle model for stability and control analysis," Multibody System Dynamics 6(2), 123-142, 2001.

[3] R.S. Sharp, "Stability, control and steering responses of motorcycles," Vehicle System Dynamics 35(4-5), 291-318. and Zeitlinger, Lisse. Vienna. 334-342, 2001. ****

[4] S. Mammar. Notes de cours de master RVSI : Automatique avancée. Université d'Evry Val d'Essonne, France. 2006.

[5] R.S. Sharp, "The stability of motorcycles in acceleration and deceleration," In: Inst. Mech. Eng. Conference Proceedings on "Braking of Road Vehicles”. MEP. London, 45-50; 1976.

[6] R.S. Sharp, "Vibrational modes of motorcycles and their design parameter sensitivities," In: Vehicle NVH and Refinement, Proc Int Conf. Mech. Eng. Publications, London. Birmingham, 107-121, 1994.

[7] H. C. Lai Liu, J.S. Lee, D.T. Wang, and L.S. Design, "parameters study on the stability and perception of riding comfort of the electrical motorcycles under rider leaning," Mechatronics, 13, 49-76, 2003.

[8] A. Modjtahedzadeh, A. and R. A. Hess, "A Model of Driver Steering Control Behavior for Use in Assessing Vehicle Handling Qualities," ASME Journal of Dynamic Systems, Measurement, and Control, Vol. 115, No. 3, 456-464, 1993.

[9] A. Bellati, V. Cossalter, S. Garbin Fac. Mechanisms of steering control of motorcycles. 9ème international conference "High-Tech Cars and Engines", ing. Univ. Di Padova, Modena, 2003.

[10] R. Lot, M. Da Lio. A Symbolic Approch for Automatic Generation of Equation of Motion of Multibody Systems. University of Padova, Italy, 2004.

[11] E. M. Kasenally, D. J. N. Limebeer and D. Perkins,"On the design of robust two degree of freedom controllers," Automatica, vol 29, No 1, 157-168, 1993.
[12] B. Amans, M. Moutreuil, RIDER ; Recherche sur les accidents impliquant un deux roues motorisé, Mars 2005.

[13] Saïd Mammar, Stéphane Espié, Static Hinf Rider for Motorcycle roll motion stabilization. ASME, American Conference on Control, juin 2006.

[14] L. El Ghaoui, F. Oustry and M. Ait Rami, "A cone complementary linearization algorithm for static output-feedback and related problems," IEEE Trans. on Aut. Cont., vol. 42, 1171-1176, 1997.

[15] Peugeot Motorcycle http://www.peugeotmotocycles.fr.

[16] H. B. Pacejka, E. Bakker and L. Lidner. A new tire model with an application in vehicle dynamics studies. in Proc. Int. Congress and Exposition, SAE paper 890087, Detroit, Michigan, 1989.

[17] H. B. Pacejka. Tire factors and vehicle handling. Int. J. Vehicle Design, 1, pp. 1-23, 1979.

[18] B. Larnaudie, S. Bouaziz, T Maurin, S. Espié, R. Reynaud, Experimental Motorcycle platform for dynamic model extraction, IEEE Intelligent Vehicles, IV2006, 2006.

[19] B. Mourillion. Notes de cours de master RVSI Estimation Filtrage et localisation. Université d'Evry Val d'Essonne, France. 2007. 